\title{
Managing Online Exams during COVID-19 Outbreak: Experience of the Centre for Quality of Assessment\& Examinations (QA Centre)
}

Mohamed, W.A.

Assistant Professor

Vice presidency for Academic Affairs Office, Centre for Quality of Assessment\& Examinations (QA Centre), Imam Abdul Rahman Bin Faisal University (IAU), KSA

\section{Introduction}

The current COVID-19 pandemic impacts on all aspects of human life including education. As a response, a growing number of higher educational institutions worldwide partially or fully shut down their campuses as a measure to contain the rapid spread of COVID-19 virus. Reports highlighted that around 1.57 billion children and youth - $90 \%$ of the world's student population were affected by the lockdown (Co-operation \& Development, 2020). Accordingly, governments rapidly deployed measures to transition courses and programs from face-to-face instruction to online delivery mode (Sahu, 2020), namely, to the usage of fully remote teaching, though there were varieties amongst institutions with regard to scope, intensity, methods (Rahim, 2020) and preparedness to face the new challenges. One of most challenging areas that required faculty to cope with was the design on online assessment and issuance of guidance (Rahim, 2020).

In Saudi Arabia, the case was no different. Since the Ministry of Health $(\mathrm{MOH})$ announced the first positive case of COVID-19 on March 2, 2020, similar precautionary actions were taken by the Saudi government to prevent the spread of COVID-19 including temporary closure of schools and universities 
(MOH, 2020). The Ministry of Education (MOE) published a guide book for university-level examination and assessment during COVID-19 Outbreak(MOE, 2020a). Based on the MOE guidance, various methods and tools of assessment were adopted to replace the face-to-face delivery mode. This goes in line with the study that identified the most prevalent e-assessment tools used for summative purposes by the American universities in 24 online courses. Results showed that written assignments (e.g., research papers, case studies and essays), discussion (discussion board, blogs, wikis) and field work (reports on collected data from field trips, visits and field training) were the most frequently eassessment tools in online courses (Kearns, 2012). Similarly, alternative online assessment methods as: assignments, projects, presentations, posters, portfolio, oral and open book exams and discussion boards were recommended to be used. However, online written exams including multiple-choice (MCQs) and essay questions were also suggested for summative assessment purposes(MOE, 2020b)

Aligned with the afore-mentioned guidance, IAU- represented by the Vice Presidency for Academic Affairs- issued guidelines for student assessment during COVID-19. Significantly, the guidelines for mid-term exams conveyed the design of online exams to ensure that they are as rigour as paper-based exams in terms of quality. Regarding the final exams, colleges were given the opportunity to decide on the online exam platform to be used: Blackboard (BB), Question Mark (QM), Zoom, Pearson, etc. Although two hours were allocated to the final exam as a maximum duration, students still had to comply with the university's regulation that they will not be allowed to log in the exam after 15 min. Only $20 \%$ of the total score (100 marks) were allocated for the final written exam. Subsequently, the academic programs had to decide on how to recalculate or distribute the rest of total marks to other alternative assessment methods mentioned earlier to be included in the continuous assessment. 


\section{Statement of the Problem}

In-spite of universities' endeavours to assess students during COVID-19 Pandemic, the management of the inevitable online delivery mode for the final written exams was not clear and put institutions at risk depending on their preparedness to bring online exams to the same academic rigour as face-to-face modality. Therefore, the overarching purpose of this paper is to document the experience of QA centre in improving the quality of online exams and ensuring academic integrity and authenticity of student performance in the exams while managing them through three main stages: pre-exams, during exams and postexams. Therefore the study tries to answer the following questions:

1. What is the suitability of using item-banking administration system as a model for online exams as perceived by students and faculty members?

2. How could online exams be efficiently managed during the COVID-19 Pandemic in terms of pre-during and post exams?

3. What are the implications for the experience of QA centre in the postCOVID 19?

\section{Aims of the study}

The current study aim to:

1. Pilot the suitability of the proposed model for using online exams at IAU as perceived by students and faculty members at a sample of two colleges.

2. Describe how to manage online exams in terms of the three stages: pre-, during and post exams during COVID-19 or other pandemics based on the QA centre experience

3. Assess the experience of using remote exam (overall assessment of the experience, advantages/best practices and challenges)as perceived by the Online Exam Committee at IAU colleges 
4. Identify lessons learned and recommendations from the QA experience to be transferred to the era of post- COVID-19

\section{Significance of the Study}

The current study has a two-fold significance. Theoretically, the study contributes to the newly conducted research in the area of assessment in pandemics and helps to direct scolars to propose models for using e-assessment as an alternate to traditional assessment. Accordingly, this contribute to the developemnt of conecptual knowledge in this area. Also, the study has applied significance as post-COVID era requires delivering assessment in online mode and ensuring its quality, accordingly through regulations and guidelines as proposed in the current study.

\section{Terms\& Definitions}

Computer-based Exams: An electronic examination, allows test activities to be carried out using different electronic platform/environment via Internet or the Intranet. The process including administration, grading, reviewing of the examination is predominantly automated(Abass, Olajide, \& Samuel, 2017)

Online Exam: A system that involves the conduct of examinations through the web or the intranet'(Ayo, Akinyemi, Adebiyi, \& Ekong, 2007). Functionally, an e-exam can be provided using a dedicated system or it can be included as a module part of a Learning Management System. Importantly, it is not necessarily to be drawn from an item-banking, though it contributes to developing an item bank through its automation processes.

Item-Banking: A Safe and sustained system to manage a relatively large number of various good items that are classified and assess a certain trait/ area in 
a way that facilitates developing equivalent forms based on certain criteria and adding and/or drawing items from the system

\section{Study Context}

It is generally accepted that assessment is at the heart of the student learning experiences and probably the drive to how students approach their learning (Rust, O’Donovan, \& Price, 2005). QA centre, one of the entities affiliated to VPAA at IAU, was established in parallel to the authentication of Guidelines for Assessment and Procedures by the IAU Council in 2017. In conformity with the vision and mission of Imam Abdel Rahman Bin Faisal University, the QA aspires to achieve excellence and leadership in developing systems and mechanisms of students' assessment and examinations nationally, regionally and internationally. The QA centre has a distinctive hierarchical organization which allows to collaborate with IAU colleges $(\mathrm{n} .=20)$ through QA units affiliated to the Vice dean for Academic Affairs. Roles and responsibilities are assigned to QA heads of units and the committee heads associated with the unit to facilitate and ensure the implementation of IAU assessment policies and procedures.

Aligned with Vision 2030 and the 'Transformation of Education in Saudi Arabia', with a shift to digital education, a key role of QA centre is to advance the automation of assessment tools and systems and ensure they are qualityembedded. In 2018, it was reported to the QA centre by heads QA units at 17 out of 20 IAU colleges that around $60 \%$ of them employ online exams for assessing their students in quizzes, mid-term and final exams using (BB). Importantly, a number of medical colleges, a large cluster at IAU, rely heavily on MCQs to assess student knowledge (Zaidi et al., 2017), and therefore, the students need to become acquainted with online assessment to ensure they prosper in their online licensure exams. However, it has been challenging that 
despite the merits of online exams (Khan \& Khan, 2019), many instructors still prefer to use deliver their exams in a paper-based mode compared to the onlinedelivery mode as they believed authenticity is potentially compromised in the latter mode (Budhai, 2020)

Most importantly, as existing assessment policies and procedures (IAU, 2017) did not comprise regulations for online exams, a radical change was needed with reference to the best practices and the thorough examination of the 'Help' section in 'BBTest'.

\section{Using item-Banking for Modelling Online Exams}

To propose a model for online exams, it was inevitable to ensure that online exams adhere to the same academic rigour as paper-based system. In doing so, alignment with learning outcomes (LOs) (Biggs, 2011), blueprinting, item analysis, providing academic integrity (Vlachopoulos, 2016) should be ensured. QA centre approached delivering online exams through the wider context of developing item banks (Dennick, Wilkinson, \& Purcell, 2009) with reference to the standards and values for assessment design that ensure its being valid, reliable, fair (Eignor, 2013), equitable, and manageable (Race, 2019) using the QM item-banking administration system.

In this context, QM administration system was investigated to check its feasibility to enable faculty members to implement assessment practices as in the assessment cycle models which direct the improvement of practices related to exams (Hassanien, 2018), in general, and to MCQ, in specific (Zaidi et al., 2017). In this framework, we proposed an examination cycle including five main stages at two levels: the item or the exam (Fig.1). It is of note to elaborate that the cycle starts with setting up the item-banking hierarchy that helps to align the created item and exams to the course learning outcomes (CLOs) and monitor the achievement of CLOs. At the item level, the item author creates the item 
using" the item card", a card with properties and information, e.g., Bloom Taxonomy, difficulty level and question types that help to classify the item in the item pool, and item bank later (Weiss, 2013). Then the item needs to be revised by peers, co-authors or expert subject matter (ESM) in terms of content and guidelines for item writing. To be published, the item should be validated by the internal reviewer, and only then, the item could be used in an exam for experimental purposes. At the exam level, the exam will be created out of the items in the "published status" and be aligned with the exam-blueprint before being delivered(administered and given to students in convenient conditions). Finally, the exam results are analysed and psychometrics of both the exam (e.g., reliability) and items (e.g., difficulty, discrimination and distractor analysis) are calculated to decide on the goodness of items and whether to be deleted, revised and modified or stored to the item-banking (Weiss, 2013; Zaidi et al., 2017).

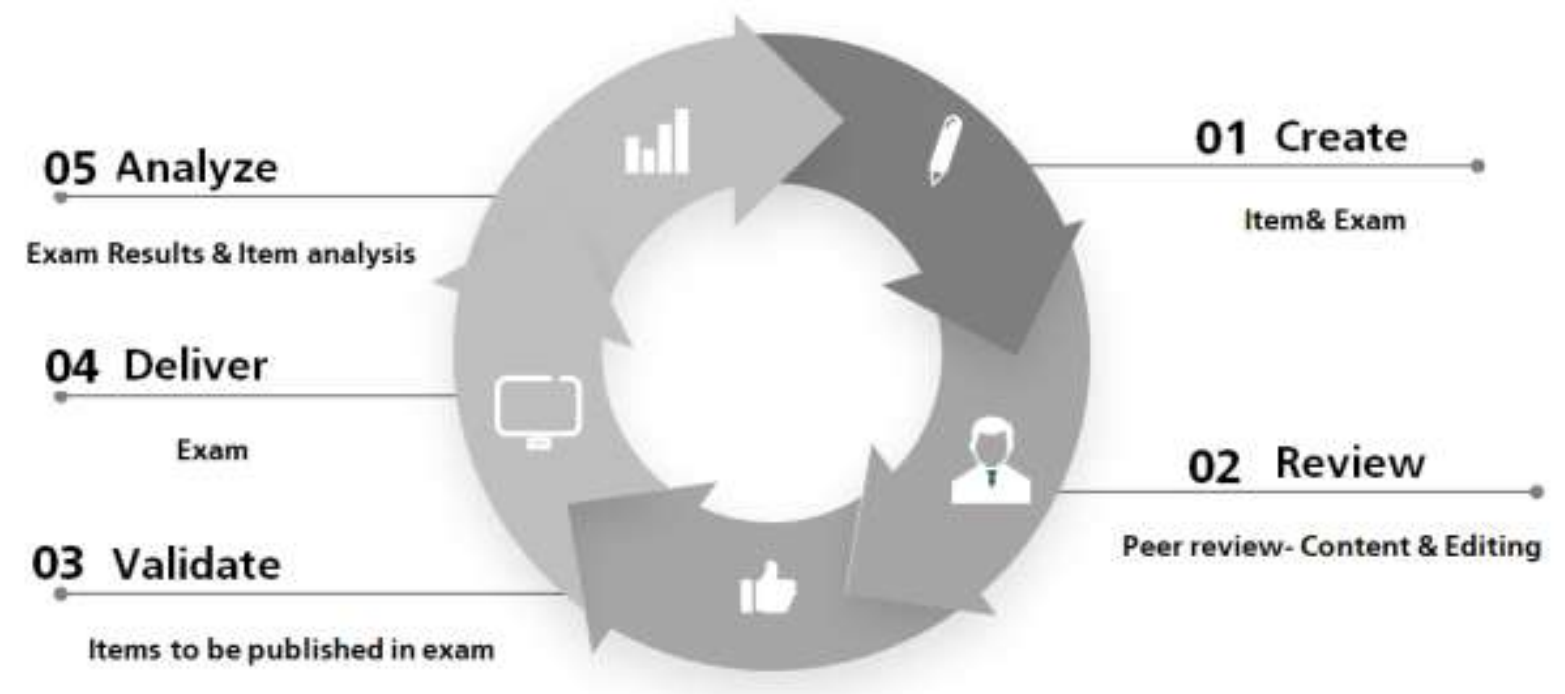

Fig. 1 Stages and processes of exam-cycle using QM administration system ${ }^{1}$ 
In addition, more features were examined to check the availability of feedback that allow students to receive instant results with correct answers and extract "coaching report" for their individual performance in relevance to Los to promote formative assessment. Also, the accessibility of assessment accommodations (AAs) for students with learning disabilities were checked to warrant an fair assessment practices, following the essence of Universal Design of Learning (Kumar \& Wideman, 2014).

\section{Piloting the model}

\section{Methodology\& Results}

During the first semester 2019-2020, the afore-mentioned new assessment practices were implemented in 19 online exams taken by 625 students, conducted in 8 courses at 7 programs, in 2 colleges following IAU assessment policies. Thirty one faculty members( $87 \%$ female), who were instructors, course coordinators, internal reviewers, heads of QA units and committees and head of departments, were trained to use the QM system and start creating item pools in their courses following the proposed model for two weeks. Four Mock exams were conducted to examine the feasibility of the system in actual testing environment at IAU computer labs. To investigate the suitability of the proposed model, a validated self-reported survey ${ }^{1}$ was constructed using the "Acceptance model" (Terzis \& Economides, 2011). The tool was given in a format of online survey to students after finishing online exams using IAU Question Pro surveys. A selection of the results is highlighted in this paper. Although 58\% of students had no or little previous experiences with online exams, $65 \%$ of students found that they were sufficiently trained on the use of online exams throughout teaching and learning process, through mock exams(42.7\%), lectures( $2.92 \%)$, assignments (12.1\%), quizzes( 21.9 ) and summative exams(20.4\%). $76 \%$ of 
students reported that online exams enabled them to get instant feedback about their performance and $72 \%$ found it helpful to review their answers. For them, online exams' easiness of use was evident as $57 \%$ and $68 \%$ expressed the easiness of interaction and easiness of use, respectively. 72. Moreover, $75 \%$ of students emphasized that course instructors helped them to use online exams and trained them. Significantly, $74 \%$ of students highlighted that online exams are related to the course content which revealed the face validity of developed online exams. Also, 61\%of students prepared well for the exam. The reported results showed how online exams were positively used for both formative and summative assessment, raising students' awareness of the privilege that they receive instant feedback which improves their learning. As per faculty, they were given an earlier validated self-reported survey after conducting a mock test using QM to check the effectiveness and easiness of the system. 83\% of faculty agreed that new practices enabled them to create online exams of a good quality in terms of blueprinting, authenticity and academic integrity. Interestingly, $66.7 \%$ of faculty agreed to generalize the new practices to the next semester. Open- ended questions asking for comments of faculty members were also included to give participants the opportunity to express themselves fully. A selection of these unprompted comments as an indication for faculty perceptions of online exams' new practices was:" enabled faculty to validate the creating exam questions as they expressed, "helped to give each student a coaching report about his/her achievement of LOs" and "ensured secured exams". The reported results for students and faculty goes in line with previous studies that demonstrated positive general impressions of students towards online exams (e.g., (Dermo, 2009; Schmidt, Ralph, \& Buskirk, 2009)

To validate whether the new practices effectively affect exam quality, a sample of "Test Score Report" were extracted and results showed that exams" reliability ranged from.64 to.89. This reflected the effectiveness of the new 
practices contributed to improving the quality of online exams and conformed with students' reports about exam face validity.

\section{Managing online exams by QA Centre}

Transfer of impact to a larger scale

Modelling online exams using item-banking administration system drastically contributed to the preparedness of IAU during COVID-19 Pandemic. With minor modifications it became an asset to maintain assessment practices with as little disruption as possible. To manage online exams during the COVID-19 pandemic, QA centre, in collaboration with VPAA, took actions and measures throughout the three stages: Pre-, during and post exams (Fig.2).

\section{Pre-exam Stage}

In this phase, regulations for ensuring the quality of online exams were issued by the VPAA to IAU colleges. Importantly was that online exams should follow the same rigour system as paper-based exams in terms of blueprinting, revision of exam paper and developing rubrics. A ProQuest survey to identify challenges while implementing the guidelines and regulations for online exams was conducted. This was to mentor the assessment practices and safeguard the authenticity and validity of student results. During Zoom meetings with heads of QA units, QA provided answers to their assessment related questions and provided constructive feedback. The outcome of this meeting was that QA issued a booklet for FAQs with solutions for each problem and challenge in both Arabic and English. For instance, to warrant academic integrity, measures for minimizing cheating as random shuffling questions and alternatives, unchecking" backtracking" to previous questions, temporarily hide feedback features from grade centre of $\mathrm{BB}$, using MCQs for assessing higher order 
thinking skills (HOTs) and allocating appropriate time for questions were announced. To validate the design and creating of an online exam, a checklist was developed, peer-reviewed and authenticated before being disseminated to IAU colleges. In addition, Online Exam Committee (OEC) was formulated in each college to supervise the administration of final online exams and report to QA centre by the end of the exams. Finally, to minimize disruptions while administering online exams using IAU different platforms, QA centre was responsible for grouping students in numbers that is compatible with the capacity of each platform.

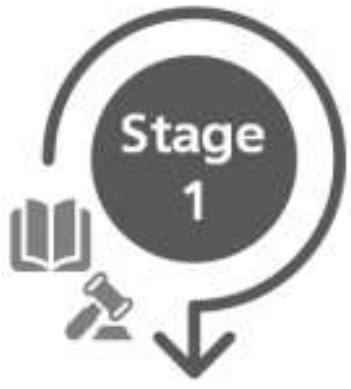

Pre-Exam

- Online Exam Cominittee

- Guidelines for online exams

- Validation of online exam quality

- Ensuring Academic Integrity

- Grouping students/Platform Capacity

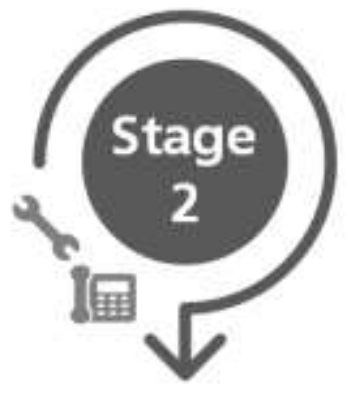

During Exam

- Rescheduling Exams

- Exam Administration

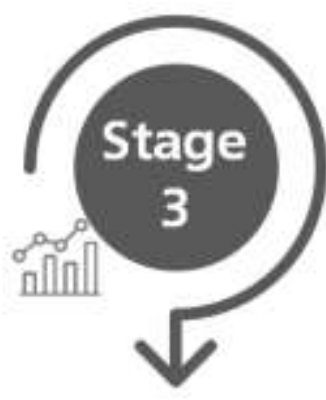

Post-Exam

Fig. 2 Stages and processes of managing online exams during COVID-19

\section{Pandemic}

\section{During-exam Stage}

As final exams were administered according to the announced schedule, the OEC became the key responsible for administering the exams in collaboration with the Deanship for E-learning\& Distance Learning. If IAU colleges were 
obliged to re-schedule any of the exams for any acceptable reason or faced with any challenge, they have to communicate with the QA centre.

\section{Post-exam Stage}

A reporting system was developed by QA centre to provide both daily and weekly reports to the VPAA. By the end of the conducted exams each day, the head of QA unit should submit an excel-format report that indicates number of test takers, successfully accomplished exams with no critical issues, number of exams per each platform and number and types of issues/problems(technical or computer/internet-related) occurred while administering the exam. By the end of each week, a collective report was submitted to the VPAA. Both the daily and collective reports that were tweeted @ Vp_academic in a format of infographic data. Importantly, more than $95 \%$ of exams were successfully accomplished with no critical issues and number of students who completed the administered exams was also above 95\% daily. More significantly, the results obtained from COEs reports for assessing the new practices indicated that $88.9 \%$ found that the overall experience was positive, successful, useful, distinct and enjoyable. $66.7 \%$ attributed the best practices to" the QA measures and procedures, esp. the validation checklist, FAQ booklet that were circulated amongst faculty" and $33.3 \%$ of colleges highlighted the use of MCQs for assessing HOTs as their best practices through the use of scenario-based questions. Additionally, online exams as a tool of technology-enabled assessment were reported to be "easily scored, cost effective, and improved students achievement" by $33.3 \%$ of colleges. On the other hand, OECs reported key challenges they faced while administering online exams during the pandemic. According to $91.6 \%$ of colleges, methods for minimizing cheating opportunities should be sought in distant exams despite the availability of "secure browsing" features in the online exam platforms. For $41.7 \%$ of colleges, using essay questions was challenging 
in terms of scoring and inserting pictures. Finally, $25 \%$ of colleges highlighted the significance of constructing item-banking and the preparedness of students with internet recourses.

\section{Conclusion}

The Bottom-line of the current study is that the "new normal" will make it imperative to leverage the power of technology -based assessment to measure what matters and invest assessment data to improve learning as it is not an end "per-se". This is only accomplished through institutional policies and governance that bring the online exams to the same academic rigour as face-toface modality. Only then, assessment disruption, due to abrupt conditions, will be kept to the minimum.

\section{Reflections \& Learned Lessons}

Opportunities: The QA centre set the roadmap to the preparedness for applying online exams based on a model that adopts developing online exams within a wider context of item-banking in a sample of IAU colleges before COVID-19 pandemic. It endeavoured to improve the quality of delivered online exams through developing guidelines, handbooks, checklists and technical support through every possible communication channel. This was accomplished through the collaboration of Heads of QA units and OECs at IAU colleges.

Challenges: Conducted distant online exams differ from online exams administered in a proctored environment. Although platforms were supported by secure browsing and QA centre set guidelines for minimizing cheating, still new methods for warranting academic integrity is required esp. with the development of new technologies of artificial intelligence (AI) and online proctoring. Lack of developed item-banking put the created items and exams to be at risk of being retired as they were disclosed to students even though 
they were randomly shuffled. Scoring essay questions is still a challenge that needs to be resolved.

Learned lessons: At the institutional scale, changes at the assessment practices may develop from the bottom up (college generated) or the top down (administrative enforced)(Oliver-Hoyo, 2011). To stay prepared, any institution should seek both channels of changes. A good example of this was transferring the impact of the pilot phase at the "college level" to the wider context of the" institutional level" and helped keeping disruptions to the minimum. A significant lesson is that commitment to standards of good assessment as validity, reliability and fairness is of no luxury since technology is merely a tool and is neither a target "per-se" nor a magic word for ensuring the quality of e-assessment.

Implications: In a new era of digitalization, students who are affiliated to generation $\mathrm{Z}$ will make it a necessity to be taught and assessed by faculty who are updated and equipped with tools of e-assessment. As there is a potential permanent shift to online learning environments, traditional assessment needs to be reconsidered. This opens new directions for adopting student-centred assessment, namely, reflection-based strategies as self and peer assessment, portfolio and the inclusion of other alternative assessment tools. As a result, a need for more faculty development programs of the "Motivation" output will be required to professionalize the faculty members to keep up with the newly adopted perspectives (Al-Eraky \& McLean, 2012). Similarly, individualized and personalized e-assessment for students who need to be accommodated should be considered to ensure the fairness of assessment 


\section{References $^{1}$}

Abass, O. A., Olajide, S. A., \& Samuel, B. O. (2017). Development of WebBased Examination System Using Open Source Programming Model. Turkish Online Journal of Distance Education, 18(2), 30-42 .

Al-Eraky, M. M., \& McLean, M. (2012). The Compass Model to plan faculty development programs. Medical Education Development, 2(1), e4-e4 .

Ayo, C., Akinyemi, I., Adebiyi, A. A., \& Ekong, U. (2007). The prospects of eexamination implementation in Nigeria. Turkish Online Journal of Distance Education-TOJDE, 8(4 . r r $\left.\varepsilon_{-}\right)$ro ، (

Biggs, J. B. T. (2011). Teaching for quality learning at university: What the student does: McGraw-hill education (UK.(

Budhai, S. (2020). Fourteen Simple Strategies to Reduce Cheating on Online Examinations. EDUCATIONAL ASSESSMENT. Retrieved from https://www.facultyfocus.com/author/stephanie-smith-budhai-phd/

Co-operation, O. f. E., \& Development. (2020). Remote online exams in higher education during the COVID-19 crisis .

Dennick, R., Wilkinson, S., \& Purcell, N. (2009). Online eAssessment :AMEE guide no. 39. Medical teacher, 31(3), 192-206 .

Dermo, J. (2009). e- Assessment and the student learning experience: A survey of student perceptions of e- assessment. British Journal of Educational Technology, 40(2), 203-214 .

Eignor, D. R. (2013). The standards for educational and psychological testing .

Hassanien, M. A. (2018). A Six-step Approach for Standardized Student Assessment in Medical Education. Journal of Advances in Medicine and Medical Research, 1-11 .

IAU. (2017). Guidelines for Policies and Procedures of Assessment and Examinations (1 ed.(.

Kearns, L. R. (2012). Student assessment in online learning: Challenges and effective practices. Journal of Online Learning and Teaching, 8(3), 198 .

\footnotetext{
${ }^{1}$ References were manged using END NOTE 7X
} 
Khan, S., \& Khan, R. A. (2019). Online assessments: Exploring perspectives of university students. Education and Information Technologies, 24(1), 661-677 .

Kumar, K. L., \& Wideman, M. (2014). Accessible by design: Applying UDL principles in a first year undergraduate course. Canadian Journal of Higher Education, 44(1), 125-147.

MOE. (2020a). Guide to Exams and Evaluation Arrangements Covid-19. Retrieved from

www.moe.gov.sa/en/HigherEducation/governmenthighereducation/Pa ges/ GuidetoEXandEV.aspx

MOE. (2020b). Guide to Exams and Evaluation Arrangements. University Semester Work during the Suspension of Education for the Prevention of COVID-19. Retrieved from https://www.moe.gov.sa/_layouts/15/MOEResp/Guide_Exams_Evalu ation_ ArrangementsEn.pdf

MOH. (2020). MOH Reports First Case of Coronavirus Infection. Retrieved from https:// www.moh.gov.sa/en/Ministry/MediaCenter/News/Pages/News-202003-02-002.aspx

Oliver-Hoyo, M. T. (2011). Lessons learned from the implementation and assessment of student-centered methodologies. Journal of Technology and Science Education, 1(1), 2-11 .

Race, P. (2019). The lecturer's toolkit: a practical guide to assessment, learning and teaching: Routledge.

Rahim, A. F. A. (2020). Guidelines for Online Assessment in Emergency Remote Teaching during the COVID-19 Pandemic. Education in Medicine Journal, $12(2$.)

Rust, C., O’Donovan, B., \& Price, M. (2005). A social constructivist assessment process model: how the research literature shows us this could be best practice. Assessment \& Evaluation in Higher Education, 30(3-r r ) ( r. $\varepsilon$. 
Sahu, P. (2020). Closure of universities due to Coronavirus Disease 2019 (COVID-19): impact on education and mental health of students and academic staff. Cureus, 12 (4 .)

Schmidt, S. M., Ralph, D. L., \& Buskirk, B. (2009). Utilizing online exams :A case study. Journal of College Teaching \& Learning (TLC), 6(8).

Slidesgo. (2020). process diagram by slidego Retrieved from Slidesgo :https://slidesgo.com/infographicscredit

Vlachopoulos, D. (2016). Assuring quality in e-learning course design: The roadmap. International Review of Research in Open and Distributed Learning: IRRODL, 17(6), 183-205 .

Weiss, D. J. (2013). Item banking, test development, and test delivery .

Zaidi, N. L. B., Monrad, S. U., Grob, K. L., Gruppen, L. D., Cherry-Bukowiec, J. R \& ‘.Santen, S. A. (2017). Building an exam through rigorous exam quality improvement. Medical Science Educator, 27(4), 793798 . 